\title{
LA CAZA DEL MUFLÓN (Ovis antiqua POMMEROL, 1879) EN EL PLEISTOCENO MEDIO DE LOS PIRINEOS: EL EJEMPLO DE LA CUEVA DE L'ARAGÓ (TAUTAVEL, FRANCIA)
}

\author{
Hervé MONCHOT \\ Laboratoire d'Anthropologie - UMR 6569 - Faculté de Médecine - Secteur \\ Nord. Bd Pierre Dramard - F 13916 Marseille cedex 20.
}

Monchot, H. 1999. La caza del muflón (Ovis antiqua Pommerol, 1879) en el Pleistoceno Medio de los Pirineos: el ejemplo de la cueva de L'Aragó (Tautavel, Francia). [Mouflon (Ovis antiqua Pommerol, 1879) hunting during the Middle Pleistocene in the Pyrenees: the example from Arago cave (Tautavel, France)]. Revista Española de Paleontología, 14 (1), 67-78. ISSN 0213-6937.

\begin{abstract}
The Arago cave (Tautavel, Pyrénées-Orientales, France) is one of the most important middle Pleistocene sites in Western Europe. Amongst the numerous faunal remains found, one species stands out for its notable rarity and originality: the mouflon. They were particulary abundant in levels $F(M N I=83)$ and $G(M N I=42)$. Using Payne's method we found mainly adults in the same layers. Furthermore, analysis of the mortality profiles indicated death by hunting. We completed these studies by estimating the weight of the population using two methods. The first one is based on the occlusal surface of the first lower molar and the second one, on the morphology of the astragalus. The frequencies of skeletal parts showed a high correlation with the bone density and this indicated differential survivorship rather than bone transport by human measured by the MGUI. Furthermore the carnivore activity (gnawing marks) on the mouflon assembly is very weak. From the patterns of bone fracturing of the long bones and butchery marks the principal steps in the preparation of mouflon for nourishment can be followed. These analyses attest of an intense exploitation of this animal by hominids (Homo erectus), essentially in an alimentary purpose, and allow us to propose the hypothesis that man was indeed a hunter and not a scavenger.
\end{abstract}

Keywords: Middle Pleistocene, mouflon, differential survivorship, fragmentation, cut marks, hunting, Arago cave.

\section{RESUMEN}

La cueva de l'Aragó (Tautavel, Pirineos-Orientales, Francia) es uno de los yacimientos más importantes del Pleistoceno Medio de Europa occidental. Entre los numerosos restos faunísticos recuperados destaca una especie por su notable rareza y su originalidad: el muflón antiguo. Son particularmente abundantes en los niveles F (NMI = 83) y G $(\mathrm{NMI}=42)$. Siguiendo el método de Payne, se encuentra que la mayor parte de Ovis antiqua son individuos adultos. También se ha realizado una estimación del peso de la población utilizando dos métodos. El primero está basado sobre la superficie oclusal del primer molar inferior y el segundo en la morfología del astrágalo. La frecuencia de las partes esqueléticas muestra una buena correlación con la densidad ósea y parece indicar que la frecuencia de los restos es más el resultado de una conservación diferencial que debido a transporte medido por IUGM. Por otra parte, la actividad de carnívoros (marcas de masticación) sobre el conjunto de huesos del muflón es débil. Las fracturas de los huesos largos y las marcas de descarnado permiten seguir las principales etapas del aprovechamiento alimenticio del muflón. Todos estos análisis muestran una explotación intensiva de estos animales por parte de los homínidos y permiten plantear la hipótesis del hombre cazador en lugar del hombre carroñero.

Palabras clave: Pleistoceno Medio, muflón, conservación diferencial, fracturación, marcas antrópicas, caza, cueva de l'Aragó. 


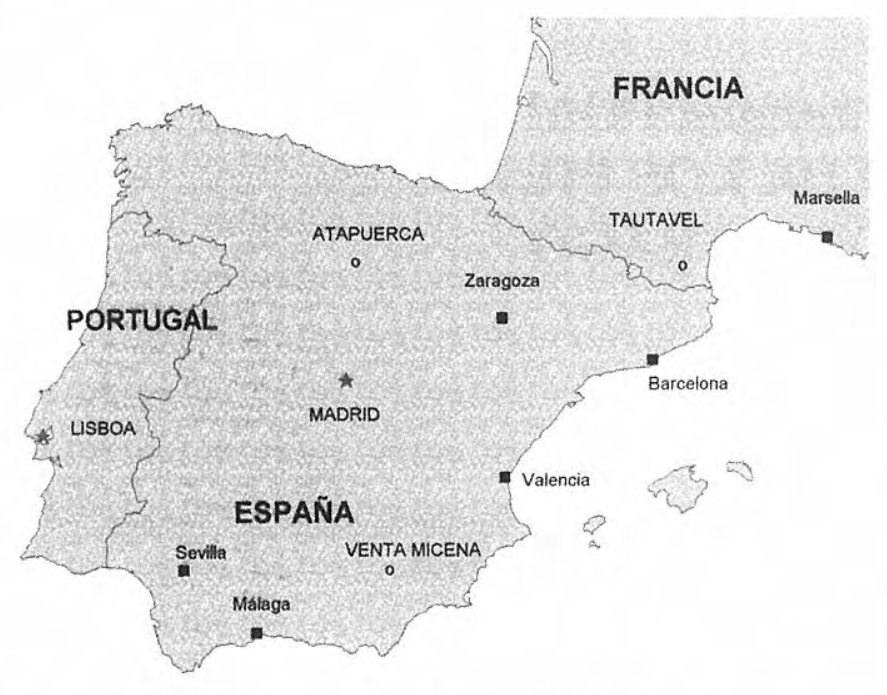

Figura 1. Localización del yacimiento de la Cueva de 1'Aragó en Tautavel (Francia) y de algunos yacimientos del Paleolítico Inferior español (o).

\section{INTRODUCCIÓN}

La cueva de l'Aragó, famosa por los numerosos restos de Anteneandertales que ha proporcionado (Lumley y Lumley, 1971), es una cavidad cárstica desarrollada en las calizas urgonienses del macizo de Corbières, en el municipio de Tautavel (Pirineos franceses), localizado a una veintena de kilómetros de Perpiñan (Fig. 1). De orientación sudeste, su entrada domina $110 \mathrm{~m}$ de un valle cerrado por donde discurre el río Verdouble.

En la actualidad se presenta como una cueva amplia, de $25 \mathrm{~m}$ de profundidad, con una anchura máxima de 10 m. La estratigrafía de los depósitos cuaternarios es conocida a partir de las secciones realizadas durante las campañas de excavación y, sobre todo, de los ocho sondeos llevados a cabo en todo el relleno (Lumley et al., 1984). El relleno cuaternario tiene un espesor máximo de 13 metros, donde se han reconocido más de 25 niveles arqueologicos superpuestos y separados entre ellos por 5 o $20 \mathrm{~cm}$ de sedimento estéril (Fig. 2). Las dataciones, efectuadas por resonancia de spin electrónico (ESR) y por isótopos de uranio (U/Th), atribuyen una edad de 700.000 años al piso estalagmítico de la base y de 92.000 años al estalagmítico final.

Los homínidos que habitaron esta cueva tuvieron una gran influencia sobre el relleno, puesto que modificaron los procesos sedimentarios y la naturaleza y aporte de elementos exocársticos, como elementos líticos, faunísticos, etc., según los niveles.

Desde 1838, fecha en la que se menciona la Cueva de l'Arago por primera vez, por el naturalista Marcel de Serres, la fauna ha sido objeto de numerosos estudios. La lista faunística actual representa una renovación de los trabajos de Crégut $(1979,1980)$. Entre las numerosas especies de macromamíferos identificadas se pueden distinguir carnívoros, cérvidos, grandes bóvidos, équidos, rinocerontes y, sobre todo, cápridos (Tab. 1).

\section{EL MUFLÓN ANTIGUO}

Entre todos los animales presentes, una especie destaca por su originalidad y su rareza: el muflón antiguo. Los estudios paleontológicos, morfológicos y osteométricos sobre el muflón de l'Aragó proporcionan datos similares a los obtenidos en la especie fósil Ovis antiqua Pommerol, 1879 y la especie actual asiática Ovis ammon (Argalis) (Crégut, 1979, Monchot, 1996a). De hecho, los Ovinae son poco comunes en el Pleistoceno. Aparecen en Europa occidental durante el Mindel (Pleistoceno Medio) aunque la presencia concreta del muflón se restringe a la franja mediterránea. Sus restos fósiles fueron descubiertos por primera vez en Pont-du-Château un lugar arenoso del Macizo Central Francés (Pommerol, 1879). Posteriormente fueron hallados también en una sima que actuaba como trampa natural para estos animales en Sauveterre-laLémance (Delpech et al., 1978), y en el noreste de los Pirineos, en la cueva de l'Aragó. Su presencia se halla también en al macizo alpino, en Uppony en Hungría (Janossy, 1969), en Italia cerca de Trieste en Visogliano (Cattani et al., 1991), y además en la región del Lazio (Pouzzolanes de Magliano; Portis, 1918). Su presencia en el Cáucaso (Baryshnikov, 1989) y en la cueva de Vallonnet está también confirmada (Moullé, 1992).

El género Ovis llega a Europa occidental procedente de Asia, donde aparece en el Villafranquiense (Ovis shantungensis Teilhard y Piveteau, 1930 ; Ovis zdanskyi Bohlin, 1937). La ruta de migración seguida por esta especie parece haber sido a través del Cáucaso, por el arco alpino, hasta el sur de Francia y los Pirineos. Estos animales, debido a razones climáticas, no pudieron sobrevivir en esta región más allá del Mindel-Riss (Crégut-Bonnoure, 1992).

En lo que concierne al ámbito de la Península Ibérica, no hay registro paleontológico de muflón (salvo con algunas dudas en Cueva Victoria, Crégut-Bonnoure, 1995). En general, los pequeños bóvidos son escasos en los yacimientos españoles del Pleistoceno Medio (Díez, 1993) y los muflones, en concreto, nunca traspasaron la barrera de los Pirineos. En este contexto, el material de la cueva de l'Aragó constituye la colección más completa e importante de la especie Ovis antiqua.

Los fósiles estudiados provienen del complejo mindeliano del relleno que consta de los niveles arqueológicos D hasta P. Estas unidades arqueologicas tienen cronología de alrededor de 450.000 años y se correlacionan con los estadios isotópicos 12 y 14.

\section{CARACTERÍSTICAS DEL MATERIAL ESTUDIADO}

El material estudiado está compuesto por 6.055 restos, que se distribuyen de la siguiente manera:

- 2.928 elementos de esqueleto craneal (2.591 son dientes aislados);

- 354 cuerpos vertebrales sin apófisis (las costillas no se han identificado debido a la posibilidad de confusión con otros taxones); 


\begin{tabular}{|c|c|c|c|c|c|c|c|c|}
\hline & 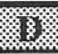 & 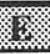 & x. & 檫 & s. & 次 & 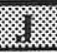 & is \\
\hline Ovis antiqua & 9 & 14 & 83 & 42 & 4 & & 7 & 4 \\
\hline Hemitragus bonali & 4 & 2 & 5 & 24 & 2 & 1 & 2 & 1 \\
\hline Rupicapra sp. & & & 1 & 1 & & & 1 & \\
\hline Capra sp. & 1 & & & & & & & \\
\hline Bison priscus & 1 & 3 & 5 & 24 & & & 1 & 1 \\
\hline Praeovibos priscus & & 1 & 7 & 13 & & & 1 & \\
\hline Dama clactoniana & & & & & 3 & 4 & 57 & 4 \\
\hline Rangifer tarandus & 1 & & 6 & 12 & 1 & 1 & 9 & 12 \\
\hline Cervus elaphus & & 1 & 10 & 27 & 5 & 5 & 43 & 2 \\
\hline Equus mosbachensis & 3 & 9 & 11 & 81 & 5 & 2 & 4 & 1 \\
\hline Stephanorhinus hemitoechus & & 1 & 3 & 33 & 5 & & 3 & 2 \\
\hline Elephas sp. & & & & 1 & & & & \\
\hline Ursus deningeri & 2 & 1 & 3 & 5 & 1 & & 2 & \\
\hline Canis lupus & & & 1 & 4 & 1 & & 2 & 1 \\
\hline Cuon priscus & 1 & 1 & 1 & 3 & & 1 & & \\
\hline Vulpes sp. & & & 2 & 2 & & & & \\
\hline Panthera spelaea & & 1 & 1 & 3 & & & 1 & \\
\hline Panthera pardus & & 1 & 3 & 1 & & & 1 & \\
\hline Felis silvestris & & & & & 1 & & 1 & \\
\hline Lynx spelaea & 1 & & 1 & 2 & & & & \\
\hline Mustela palerminea & & & 1 & 3 & & & & \\
\hline Meles meles & & & & 1 & 1 & & & \\
\hline
\end{tabular}

Tabla 1. Representación del NMI de los grandes mamíferos de la cueva de l'Aragó en los niveles arqueológicos D a K.

- 1.148 elementos de la cintura escapular y del miembro anterior (escápula, húmero, radio, ulna, carpales, metacarpo y falanges);

- 1.036 elementos de la cintura pélvica y del miembro posterior (coxal, fémur, tibia, rótula, maleolar, tarsales, metatarso y falanges);

- 589 sesamoideos.

El Número Mínimo de Individuos (NMI) cuantificado de muflones antiguos es de 192, de los cuales 56 son inmaduros y el resto adultos (136). Estos datos se han obtenido a partir de una combinación de material dentario, que en la mayoría de los niveles se trata del D4 y del M3. La distribución por niveles arqueologicos es de 83 en el nivel F y 42 en el nivel G (Fig. 3).

En los niveles $F$ y $G$, se encuentran todos los elementos anatómicos que componen un esqueleto (Fig. 4). La diferencia más significativa observada entre ambos niveles (Kolmogorov-Smirnov $Z=1,634$; $\mathrm{p}<0,01$ ) se explica únicamente por la intensidad de la fragmentación de los huesos. Esto se confirma por el índice de destrucción de los huesos (Dientes/Número de Restos) que es de $49 \%$ en el nivel F y de 30,4\% en el nivel G. Se han encontrado muy pocas conexiones anatómicas in situ, excepto algunos carpales, tarsales y vértebras.

A diferencia de los numerosos yacimientos del Paleolítico Inferior y Medio del sur de Francia, como por ejemplo Lazaret (Valensi, 1996), Lunel-Viel I (Fosse, 1996) o de España, como Galería en la Sierra de Atapuerca (Huguet et al., en prensa), Venta Micena (Palmqvist et al., 1996), o Cova Negra (Villaverde et al.,
1996), los restos de muflón están muy poco afectados por los carnívoros $(0,4 \%)$. En los huesos no se encuentran huellas de surcos, improntas o depresiones efectuadas por los predadores (Miller, 1994). Ocasionalmente se han observado cúpulas de pequeño diámetro atribuibles a un carnívoro de tamaño mediano.

A pesar de existir una diversidad importante de carnívoros en la cueva de l'Arago, estos animales no son muy abundantes. Se han documentado tan solo 59 individuos que contrastan con los 662 ungulados identificados (niveles D a K, excepto en el nivel FG). Por tanto, se obtiene una relación NMI carnívoros / NMI (carnívoros + ungulados) de $8 \%$. Esta cifra se adecua a las obtenidas en yacimientos arqueológicos donde los carnívoros representan por regla general menos del $10 \%$ y raramente superan el $13 \%$ del NMI total (Klein y CruzUribe, 1984). Esto se explica, según los autores, por la tendencia que tienen los homínidos y los carnivoros de evitarse mutuamente para escapar de conflictos eventuales.

Citemos por comparación, los yacimientos con el porcentaje de carnívoros siguientes: Camiac, que es un cubil de hienas $=21 \%$ (Guadelli, 1989); la Nauterie, osera $=64 \%($ Simonet, 1990); Venta Micena $=13 \%$ (Palmqvist et al., 1996); Galeria nivel TG10B $=11,9 \%$ (Huguet et al., en prensa), Lunel-Viel, cubil de hienas = $20 \%$ (Fosse, 1996). En el yacimiento de Le Lazaret, hay una relación del $28 \%$, pero en este caso estamos ante una acumulación antrópica con explotación de carnívoros (Valensi, 1996). 


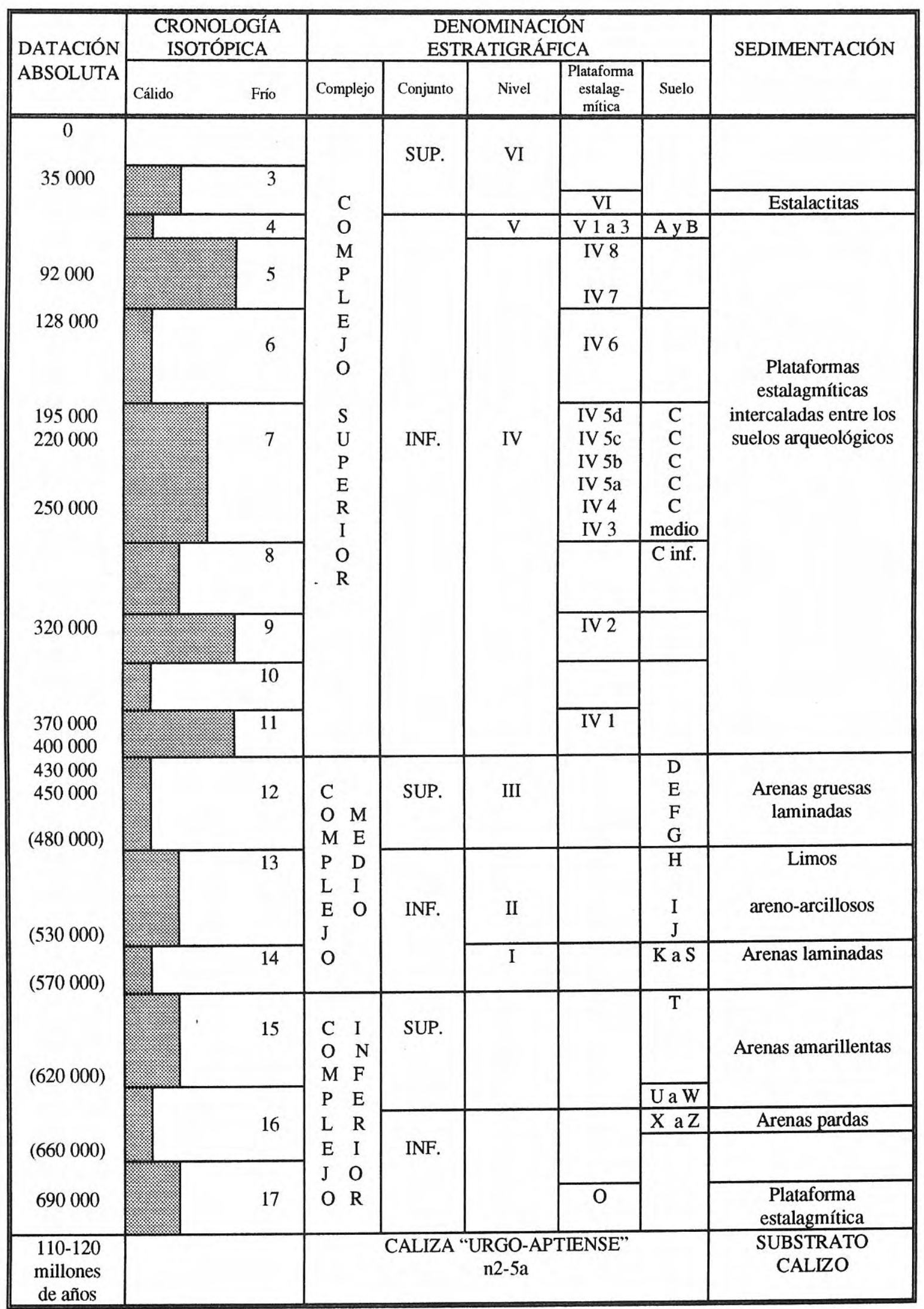

Figura 2. Estratigrafía del relleno de la cueva de l'Aragó (extraído de Lumley et al., 1984).

Una acción secundaria de los carnívoros sobre las acumulaciones óseas es posible (ocupación mixta) pero difícil de evidenciar en un yacimiento fuertemente antropizado como el de Aragó. 


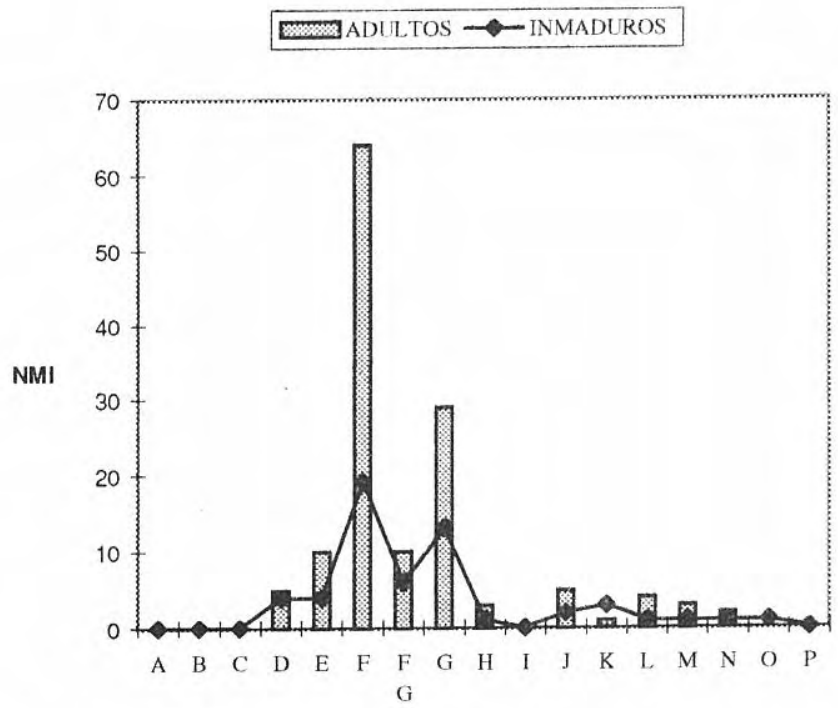

Figura 3. NMI del muflón de los niveles arqueológicos A a P.

\section{PERFIL DE MORTALIDAD}

Existen dos tipos teóricos de perfiles de mortalidad que en zoología se han denominado perfil catastrófico y perfil atricional o de desgaste. El primero de ellos se caracteriza porque las sucesivas clases de edad van poseyendo cada vez menos individuos. El otro perfil es el resultado de una mortandad que afecta principlamente a los miembros más débiles de una población, es decir a los infantiles y progresivamente menos juveniles, adultos y seniles (Levine, 1983; Blasco Sancho, 1995).

La estimación de la edad de los animales ha sido establecida a partir del desgaste de la superficie oclusal de los dientes inferiores, tanto para los deciduales como para los definitivos. Estos datos han sido elaborados a partir del sistema de esquematización de Payne (1973, 1987). Así, hemos hecho una primera clasificación basada en la observación de los estadios de erupción dental de los fragmentos mandibulares $(\mathrm{NR}=164,93$ derechos y 71 izquierdos), y después sobre el grado de desgaste de la superficie oclusal. Los M1 han sido tomados como referencia y la clasificación ha sido refinada utilizando los M2, depués los D4, M3 y siguientes. De esta forma los dientes aislados pueden ser integrados en el análisis por comparación.

Se han definido así nueve estadios de desgaste (Fig. 5). No queda más que establecer las correspondencias entre las series de piezas clasificadas por orden de edad gracias a una escala numérica (ovinos domésticos). Este método ha sido preferido al que consiste en medir la altura de las coronas dentarias (Klein et al., 1983; Klein y Cruz-Uribe, 1984).

Los perfiles obtenidos en los niveles F y G muestran una clara sobreabundancia de adultos jóvenes (categorías E-F, Fig. 5). El número de adultos identificados en este tipo de análisis, permite diferenciar, en el caso del muflón, entre una población natural y una población cazada por el hombre (Pfeffer, 1967). En una población natural, la mortalidad se da principalmente entre los animales jóvenes y seniles, mientras que, en una población sometida a la caza, las perdidas de ejemplares adultos son importantes. La categoría D es la única que presenta individuos inmaduros. Esto puede ser debido a la gran fragilidad de los dientes deciduales, los cuales son más sensibles a la destrucción diagenetica. Hay que señalar, no obstante, que la mortalidad infantil es muy importante, y que los individuos inmaduros no son presas habituales de los cazadores humanos actuales. Por sł parte, los animales seniles rehuyen con más facilidad la presencia humana debido a su mayor experiencia (Pfeffer, 1967).

En general, estos perfiles de mortalidad se distinguen netamente de los causados por diversos carnívoros (cánidos, félidos, hiénidos) en los que se observa un dominio de los individuos juveniles y seniles (Klein, 1982; Fosse, 1996; Palmqvist et al., 1996).

Los perfiles de mortalidad muestran una actividad cinegética y se distinguen bien del perfil de mortalidad de tipo catastrófico y del perfil de muerte natural (Lyman, 1987a; Stiner, 1990). Stiner (1994) considera que los grupos humanos parecen ser los únicos agentes depredadores que regularmente producen patrones de mortalidad sesgados hacia los adultos (en diferentes conjuntos de presas). Así, la presencia de individuos jóvenes está evidenciada igualmente por la presencia de numerosas epífisis no soldadas. Finalmente, señalemos que en estos mismos niveles arqueológicos (F y G), los cérvidos jóvenes (Cervus elaphus y Rangifer tarandus) son muy poco numerosos, la predación se ha realizado sobre todo en los individuos adultos (Moigne, 1983; Pernaud, 1990).

\section{ESTIMACIÓN DEL PESO}

La estimación del peso de una presa es importante por una parte desde el punto de vista paleoecológico para la elaboración de los cenogramas y por otra a fin de calcular la contribución alimenticia que corresponde a cada una de las diferentes especies de mamíferos cazados. Hay numerosos trabajos basados sobre las leyes de alometría que permiten estimar el peso de los animales fósiles (Damuth, 1981; Scott, 1983).

Se ha efectuado mediante dos métodos distintos una aproximación al peso del muflón fósil: el primero utiliza la superficie del M1 (Legendre, 1988), mientras que el segundo utiliza la superficie del astrágalo (Martinez, 1991). La aplicación del primer método ha proporcionado los siguientes índices:

$\mathrm{P}=112 \mathrm{Kg}(13 \mathrm{Kg}$ en 111 dientes (C.V. = 15.644; Min. $=71 \mathrm{Kg}$ y Max. $=156 \mathrm{Kg}$ ).

El segundo método ha proporcionado los siguientes resultados:

$\mathrm{P}=128 \mathrm{Kg}(12 \mathrm{Kg}$ en 36 astrágalos (C.V. = 9.743; Min. $=108 \mathrm{Kg}$ y Max. = $156 \mathrm{Kg}$ ).

La comparación de ambos métodos muestra que la estimación del peso a partir de los parámetros dentarios es baja. Esto se atribuye en gran parte a la edad de los animales, que se reconoce a través de los estadios de 


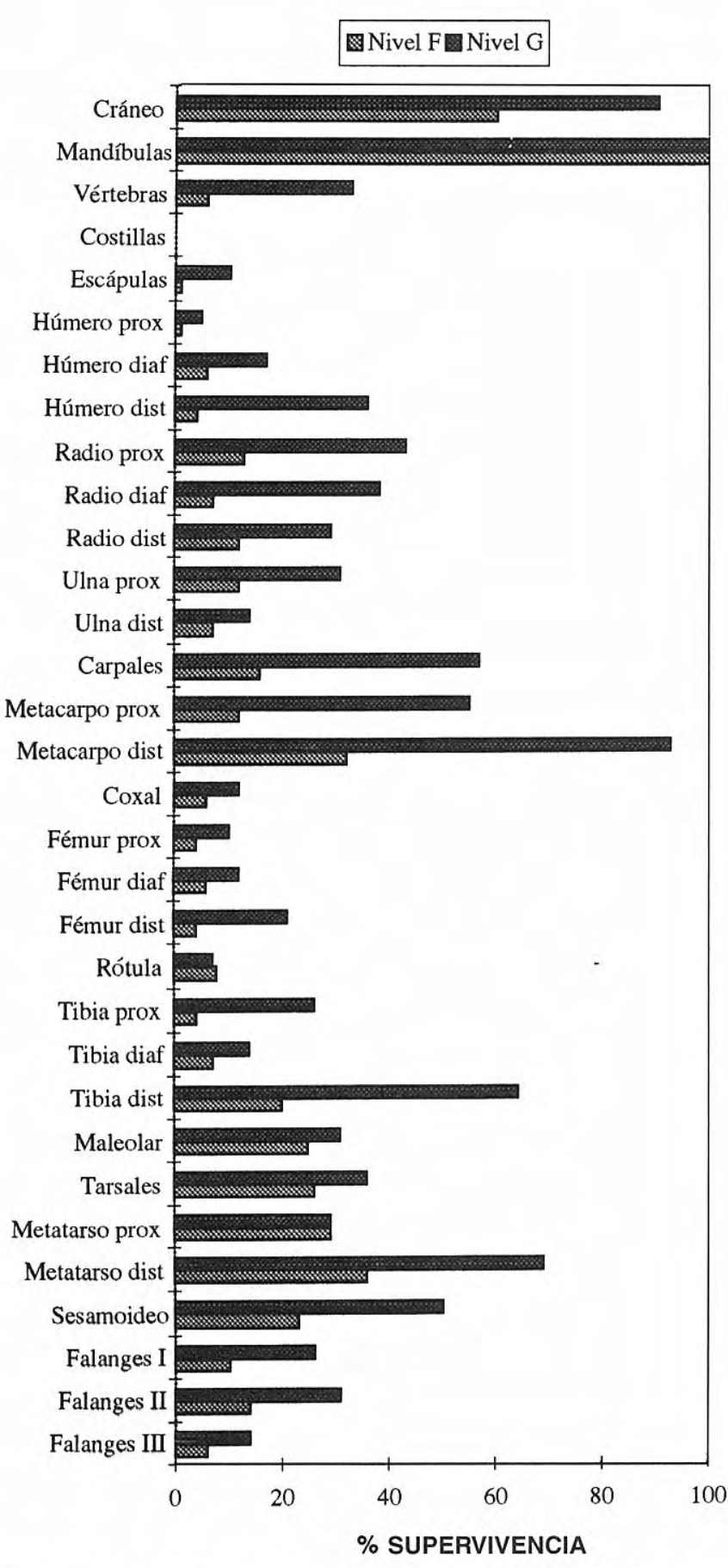

Figura 4. Porcentaje de supervivencia (\% UAM = Mínimo de Unidades Anatómicas) de los diferentes elementos anatómicos de los niveles F y G.

desgaste de los dientes. De este modo se constata que los animales más viejos (estadios 9 y 14 del esquema de Payne) son también los menos pesados. Esto es atribuible al hecho de que la superficie de los molares inferiores, y en particular la del M1 disminuye en función de la edad, así como la altura de las coronas dentales. Paralelamente, no hay que olvidar que los individuos seniles presentan fuertes pérdidas de peso ligadas a una peor alimentación debido a la deterioración de la dentición (Pfeffer, 1967).
Además, los datos que proporciona esta estimación media (112 Kg y $128 \mathrm{Kg}$ ) pueden parecer bajos en relación con la especie actual (Ovis ammon). Hoy en día, los grandes muflones machos de l'Altaï pueden sobrepasar los $200 \mathrm{Kg}$, pero los machos del Pamir pesan entre 110 y $160 \mathrm{Kg}$ y sus hembras oscilan entre los 90 y los $100 \mathrm{Kg}$.

\section{CONSERVACIÓN DIFERENCIAL VERSUS TRANSPORTE DIFERENCIAL}

Una de las maneras de valorar la posibilidad de un transporte diferencial de partes anatómicas en un yacimiento es utilizar los índices de utilidad general (Binford, 1978) que tiene como objetivo proveer de datos cuantitativos sobre el valor nutritivo potencial de cada parte esquelética (carne, grasa, médula, etc.) Este índice ha sido utilizado por algunos especialistas de zooarqueología bajo la premisa de que las partes más útiles son las que el hombre decide llevarse al campamento (Speth, 1983), mientras que otros autores lo han criticado o modificado (Lyman, 1985; Metcalfe y Jones, 1988; Jones y Metcalfe, 1988).

Por lo tanto, si nos referimos al comportamiento de elementos anatómicos en los niveles F y $\mathrm{G}$ en función de los IUGM (Índice de Utilidad General Modificado), observamos una representación del tipo Reverse (bulk) utility butchering strategy (curva en L, Fig. 6). Esto se traduce en una baja frecuencia de elementos con un alto valor energético y una elevada frecuencia de elementos de débil potencial energético (Binford, 1978).

La conservación diferencial nos permite explicar la razón de la ausencia de ciertas partes anatómicas. De hecho, se nota que las partes que faltan o que están infrarrepresentadas (esencialmente las partes proximales de los huesos largos, tibia y húmero), son las partes menos densas, las más ricas en tejido esponjoso, y las que quedan habitualmente sometidas a una destrucción más rápida.

Por ejemplo, el cálculo del NME ha proporcionado, en el caso del húmero, 47 extremidades distales y 7 extremidades proximales. El mismo fenómeno se produce en la tibia, donde se han reconocido 22 fragmentos proximales y 131 distales. Un transporte selectivo desde el lugar de obtención hasta la cueva no puede ser deducido de ésto, ya que es poco probable que los homínidos hubieran trasladado sólo las partes distales del húmero o de la tibia. Parece pues que la pobre representación de las extremidades proximales de húmero y tibia es debida a una conservación diferencial y al sesgo producido en el proceso de la fracturación antrópica. Este fenómeno afecta intensamente a las partes menos densas del hueso, dando lugar a fragmentos relativamente pequeños y, a menudo, indeterminables.

De este modo, si elaboramos un gráfico para los restos del muflón del nivel $\mathrm{G}$, donde se represente la abundancia de los elementos anatómicos (en MNI) en función de su densidad (Lyman, 1984), observamos que se da una buena correlación $(\mathrm{r}=0,791 ; \mathrm{t}=5,916 ; \mathrm{p}=0,0001)$ y que 
los elementos más densos son los más abundantes (Fig.7). Señalemos que si utilizáramos el UAM (Unidad Animal Mínima, Binford, 1984) como unidad de descuento para los elementos anatómicos, habríamos obtenido una correlación mejor $(\mathrm{r}=0,841 ; \mathrm{t}=7,111$; $\mathrm{p}=0,0001)$. Señalemos igualmente la correlación negativa entre los IUGM y el potencial de destrucción de cada hueso con relación a su densidad (Tau de Kendall $=-0,275 ; \mathrm{p}=$ 0,0399).

En cuanto a los resultados, llegamos a la conclusión de que una curva de utilidades en una población arqueológica en forma de L, medido por los IUGM, muestra que la representación esquelética, sobre todo en un medio cárstico, es más el producto de una destrucción diferencial que el efecto de un transporte ligado a los homínidos (Lyman, 1985, 1992; Grayson, 1989). La presencia de diversas partes anatómicas en un yacimiento no sólo está condicionada por las decisiones de transporte de la presa, sino también por las capacidades de fracturatión de los huesos por sus depredadores y por la destrucción selectiva como consecuencia de ciertos procesos naturales que se producen en el lugar donde los desperdicios han sido enterrados.

\section{FRACTURACIÓN}

Cabe destacar que todos los huesos largos están fragmentados, así como los huesos del cráneo, las mandíbulas, la pelvis, los calcáneos y las primeras falanges. El estudio de la fracturación de los huesos largos de muflón forma parte de un trabajo precedente (Monchot, 1997), que se puede resumir en los siguientes puntos:

- Solamente se han recuperado dos huesos largos completos: una tibia y un fémur pertenecientes al nivel $\mathrm{G}$.

- Según el método desarrollado por Richardson (1980), es posible distinguir dos grupos conforme al comportamiento de los extremos articulados: el primero está constituido por la tibia y el húmero, que muestran un déficit en un extremo articular, con una frecuencia próxima (\% diff. > 70). El segundo muestra que unos huesos (radio, fémur, metápodos) tienen una diferencia menor entre los dos extremos articulares (\% diff. <30).

- La asociación circunferencia-longitud de la fractura en la zona de la diáfisis muestra que la mayoría de fragmentos $(69 \%)$ son cortos y poseen una circunferencia inferior a la mitad de la original, que es característica de una acción antrópica.

- Finalmente, el estudio morfológico de las fracturas de los huesos largos muestra un predominio de las fracturas de tipo curvado o espiraliformes (38\%) y un ángulo de fractura muy oblícuo (36 \% de los restos). Esto indica que la fracturación de los huesos largos, en la mayoría de casos, se produce en estado fresco, y que son los homínidos, y no la presión de los sedimentos, su responsable principal (Villa y Mahieu, 1991). Además, la mayoría de los bordes de fractura son lisos (48 \%), lo que tiende a mostrar que la fracturación ha sido provocada por una fuerza dinámica, como un golpe y no por una

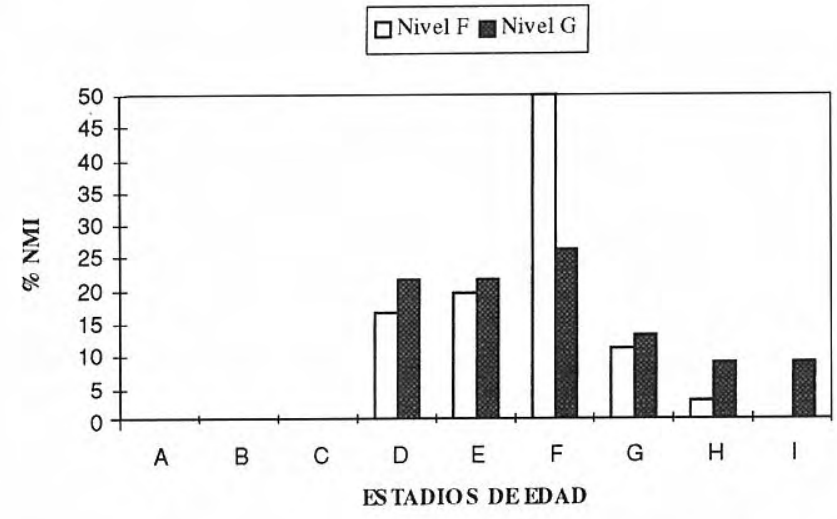

Figura 5. Perfiles de mortalidad del muflón en los niveles $\mathrm{F}$ y $G$ (método de Payne). Estadios A a D = inmaduros; $\mathrm{E}-\mathrm{F}-\mathrm{G}=$ adultos; $\mathrm{H}-\mathrm{I}=$ seniles. Estadio A = D4 acaba de salir (0-2 meses); Estadio B = aparición del M1 (2-6 meses); Estadio $\mathrm{C}=\mathrm{M} 1$ en la tabla de oclusión, aparición del M2 (6-12 meses); Estadio D = M2 en la tabla de oclusión, aparición del M3 (1-2 años); Estadio $\mathrm{E}=$ aparición del lóbulo distal del M3 (2-3 años); Estadio F = lóbulo distal del M3 en la tabla de desgaste (3-4 años); Estadio G = el M2 presenta una superficie de desgaste única (4-6 años); Estadio $\mathrm{H}=$ M3 plano y muy desgastado (6-8 años); Estadio $\mathrm{I}=$ molares muy desgastados, a menudo hasta la raíz (8-10 años).

fuerza estática, similar a las que se producen durante el enterramiento o por las mandíbulas de un carnívoro.

\section{MARCAS ANTRÓPICAS}

En primer lugar, es necesario definir ciertos términos y conceptos, puesto que palabras como la "carnicería" tienen distintos significados según los autores. Así, la "carnicería" puede precisarse como una actividad antrópica relacionada con la modificación y la reducción de una presa en partes utilizables. El sentido de "utilizable" designa todos los productos de un animal muerto, sin restringirse al alimento de la carne. No hay que considerar el tratamiento del animal muerto como un acto sencillo, sino como una serie de actividades directamente ligadas a la extracción de recursos: piel, pelos, tendones, ligamentos, huesos, cuernos, médula, grasa, sangre, vísceras, carne,...

El hombre provoca, mediante su acción, distintas señales sobre los huesos. Sin embargo, numerosos animales (roedores, hienas, carnívoros en general) dejan marcas parecidas durante la explotación de sus presas (Blumenschine et al., 1996). Para calificar una señal de antrópica, hay que reunir dos criterios (Lyman, 1987b; 1994):

- debe haber una reiteración de marcas parecidas, tanto en su posición como en su disposición sobre los esqueletos (redundancy criterion);

- debe existir un condicionante anatómico (purposiveness criterion). 


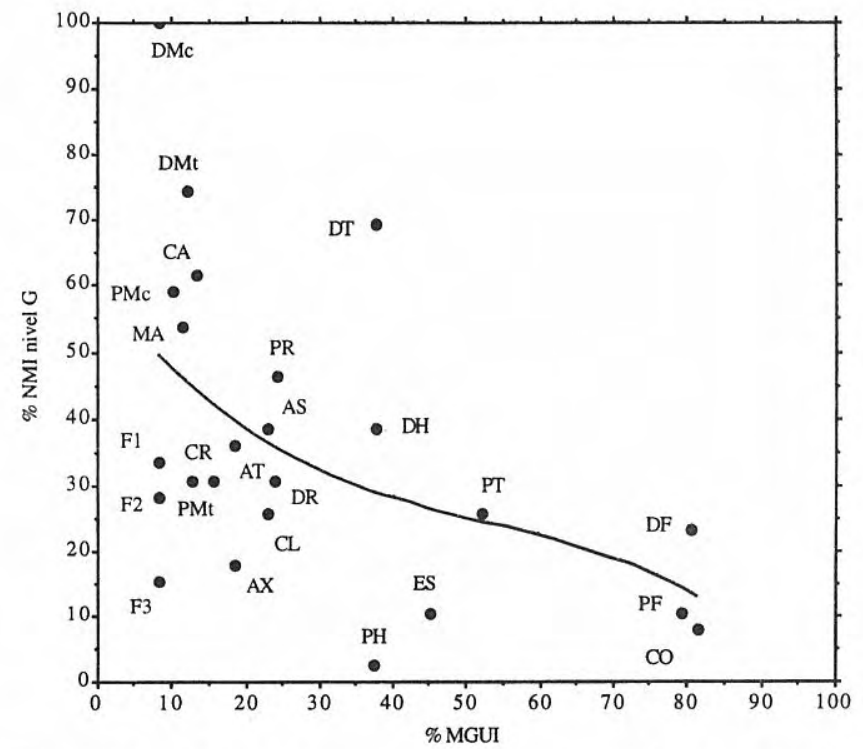

Figura 6. Relación entre \% MGUI (segun Binford, 1978) y el \% NMI en el nivel G. (AS = astrágalo; $\mathrm{AT}=$ atlas; $\mathrm{AX}=$ axis; $\mathrm{CA}=$ carpales; $\mathrm{CL}=$ calcáneo; $\mathrm{CO}=$ coxal $; \mathrm{CR}=$ cráneo; $\mathrm{DF}=$ Fémur dist.; $\mathrm{DH}$ = húmero dist.; DMc = metacarpo dist.; DMt = metatarso dist.; $\mathrm{DR}=$ radio dist.; $\mathrm{DT}=$ Tibia dist.; $\mathrm{ES}=$ escápula; $\mathrm{F} 1$ = falange $1 ; \mathrm{F} 2$ = falange $2 ; \mathrm{F} 3$ = falange $3 ; \mathrm{MA}=$ mandíbula $; \mathrm{PF}=$ fémur prox.; $\mathrm{PH}=$ húmero prox.; $\mathrm{PMc}=$ metacarpo prox.; $\mathrm{PMt}$ = metatarso prox.; $\mathrm{PR}=$ radio prox.; $\mathrm{PT}=$ tibia prox.)

Así, para cada elemento esquelético intervenido se ha hecho una descripción precisa de las señales observadas, teniendo en cuenta su localización, su orientación en el fragmento, su frecuencia y su morfología (Binford, 1981; Bunn, 1982; Lyman, 1987b; Valensi, 1991). La interpretación de las estrías se basa en la literatura arqueológica y etnográfica (Trolle-Lassen, 1990; Alhaique, 1994; Capaldo, 1997). Los resultados han sido comparados con los obtenidos por Binford (1981) sobre los esquimales Nunamiut, utilizando su misma codificación.

Sobre 2.755 huesos de muflón (dientes aislados y sesamoideos excluidos), 298 presentan incisiones (el $10,81 \%$ del material, Fig. 8). La frecuencia en la presencia de marcas de corte sobre los distintos elementos del esqueleto no parece ser casual (ZK.S. = 2,847; $\mathrm{p}<0,0001)$. De este modo, la frecuencia de estas incisiones sobre cualquier elemento del esqueleto no se explica por su abundancia, y esto es probablemente

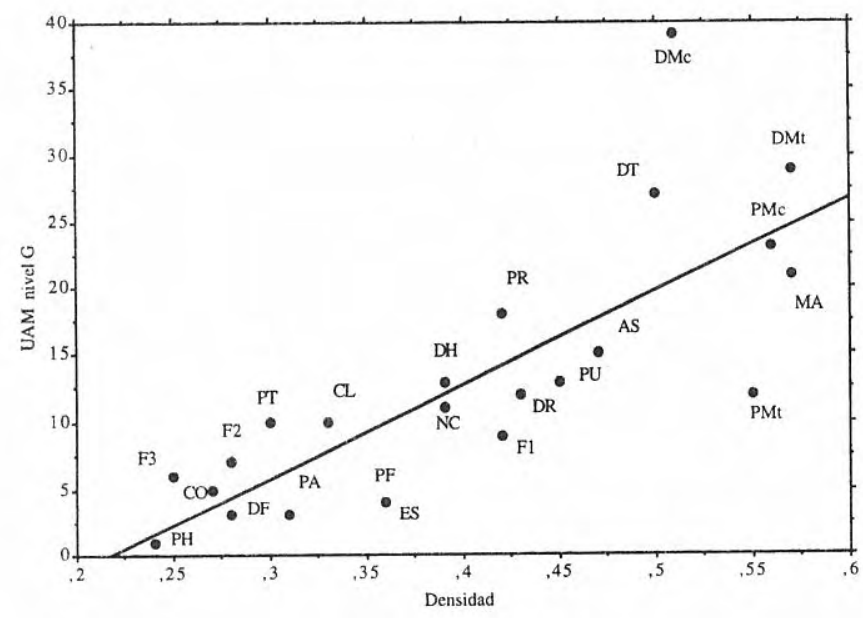

Figura 7. Relación entre la densidad (segun Lyman, 1984) y el UAM (segun Binford, 1984) en el nivel G. (AS = astrágalo; $\mathrm{CA}=$ carpales $; \mathrm{CL}=$ calcáneo; $\mathrm{CO}=$ coxal; $\mathrm{CR}=$ cráneo; $\mathrm{DF}=$ Fémur dist.; $\mathrm{DH}$ = húmero dist.; $\mathrm{DMc}=$ metacarpo dist.; $\mathrm{DMt}=$ metatarso dist.; DR = radio dist.; DT = Tibia dist.; $\mathrm{ES}=$ escápula; $\mathrm{F} 1$ = falange $1 ; \mathrm{F} 2$ = falange $2 ; \mathrm{F} 3$ = falange $3 ; \mathrm{MA}=$ mandíbula $; \mathrm{NC}=$ navicular; $\mathrm{PA}=$ patella; $\mathrm{PF}=$ fémur prox.; $\mathrm{PH}=$ húmero prox.; PMc $=$ metacarpo prox.; $\mathrm{PMt}=$ metatarso prox.; $\mathrm{PR}=$ radio prox.; $\mathrm{PT}=$ tibia prox.; $\mathrm{PU}=$ Ulna prox.)

debido a las diferencias en el tratamiento de las distintas partes del animal. Estas marcas tienen relación con las exigencias de una "matanza sistemática" (Bunn y Kroll, 1986).

Los elementos que llevan incisiones se hallan en todos los niveles arqueológicos, del nivel $\mathrm{D}$ al $\mathrm{P}$, representando un mínimo de 27 individuos a lo largo del relleno. Esta distribución es proporcional al número de piezas existentes en cada nivel y no hay diferencias estadísticas entre los niveles arqueológicos (ZK.S. = 1,004; p > 0,1). Las únicas variaciones observadas parecen debidas al azar.

El análisis estadístico subraya que las incisiones afectan tanto a los elementos derechos como a los izquierdos (ZK.S. = 1,297; p = 0,1946). Eso quiere decir que ambos lados del animal han sido tratados de manera parecida, sea cual sea el nivel arqueológico.

En el caso de los huesos largos, las incisiones se hallan sobre todo en las metáfisis y epífisis (80\%). Las diferencias observadas en el tipo de hueso (Tab. 2) no son el resultado de la elección, sino el resultado de la

\begin{tabular}{|c|c|c|c|}
\hline & PROXIMAL & DIAFISIS & DISTAL \\
\hline Húmero & 0 & 33 & 67 \\
\hline Radio-Ulna & 68 & 13 & 19 \\
\hline Fémur & 36 & 50 & 14 \\
\hline Tibia & 0 & 37 & 63 \\
\hline Metápodo & 63 & 0 & 37 \\
\hline
\end{tabular}

Tabla 2. Frecuencia de marcas de corte sobre las diferentes zonas de los huesos largos. 
conservación diferencial. En el caso de las caras encontramos algo parecido. La situación de las estrías domina en una sola cara; por ejemplo, para los extremos distales de la tibia, los cortes se encuentran esencialmente sobre la cara medial ( $52 \%$, el otro $48 \%$ se reparte entre las otras tres caras). Estas señales son transversales $(31,6$ $\%)$ u oblicuas $(68,4 \%)$. En el caso del húmero, el 83,3\% de las marcas se encuentran sobre la cara medial, pero no se encuentra ninguna estría transversal. En el radio, las estrías se localizan sobre todo en la cara interna (58\%). Finalmente, ante las pocas señales encontradas sobre los extremos del fémur no se puede resaltar ninguna organización concreta.

La fragmentación es muy importante y no permite definir un tipo de organización preferencial para las marcas de corte situadas sobre las diáfisis. Las estrías son oblicuas $(47,6 \%)$ o transversales $(42,8 \%)$. Sea cual sea el tipo de hueso, estas señales se sitúan tanto en las caras anteriores $(38,1 \%)$ como posteriores $(45,2 \%)$. Esto es debido en gran parte a la anatomía de los huesos, así como a la determinación diferencial. Las señales localizadas sobre la zona central de las diáfisis son escasas. Lo contrario se ha observado sobre el taxón Capra ibex de la cueva des Églises (Delpech y Villa, 1993). No se ha observado ninguna organización transversal preferencial de las estrías, como las que se reconocen cuando son motivadas por el pisoteo (Olsen y Shipman, 1988).

Estas incisiones se parecen a las observadas en numerosos yacimientos, como son las de las cabras de la Cueva des Églises (Delpech y Villa, 1993), los cérvidos de la Cueva de Lazaret (Valensi, 1991), la acumulación ósea de la Cueva de Gabasa 1 (Blasco Sancho, 1995) y los yacimientos de la zona sur del Mediterráneo español (Pérez Ripoll, 1992). El análisis de todas las marcas de corte nos ha permitido distinguir varias etapas en el proceso de aprovechamiento del animal (Monchot, 1996b):

- El despellejamiento: las señales localizadas de este tipo muestran con certeza una acción destinada a la extracción de la piel. Son las que se han encontrado en los cráneos, las mandíbulas, las falanges y los extremos de los metápodos. Es importante subrayar que hay que distinguir entre dos tipos de despellejamiento: uno se efectúa sobre el animal entero, con objeto de recuperar la piel, mientras que el otro se considera como una etapa sencilla, necesaria antes de la descarnación, que se pueden haber practicado sobre un animal descuartizado.

- La descuartización: la separación de la cabeza del esqueleto axial se confirma por la presencia de dos restos que muestran incisiones a nivel del cóndilo occipital. El cuarto anterior se obtiene generalmente al separar la escápula de la caja torácica. Sin embargo, ninguna estría que denote esta acción ha sido identificada con certeza. Para el miembro posterior, se ha evidenciado la desarticulación coxo-femoral, pero no tenemos ninguna prueba de la desarticulación sacro-coxal.

- La desarticulación: a esta actividad se le han atribuido la mayoría de las señales. Se han producido, en general, unas señales cortas, finas, poco profundas y transversales al eje longitudinal de los huesos largos.

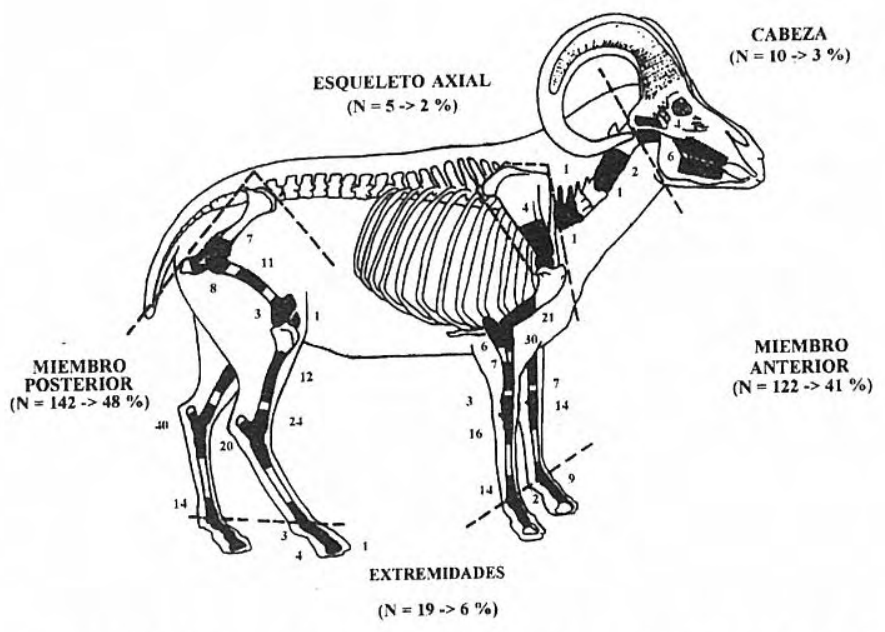

Figura 8. Repartición de las marcas de corte sobre el esqueleto de muflón.

Ninguna marca confirma con certeza la desarticulación de la mandíbula. No obstante, estos restos muestran que las cabezas estaban en parte explotadas por los homínidos para sacar la lengua y los músculos de la mejilla. La fracturación extrema no permite evidenciar la desarticulación costilla-vertebra. Cabe destacar la presencia de grupos pequeños de vertebras en conexión anatómica, sobre todo las vértebras cervicales, que atestiguan una segmentación voluntaria del esqueleto axial en unidades más reducidas. La desarticulación del miembro anterior se lleva a cabo a dos niveles: en el codo y en los carpales. Para el miembro posterior, hemos evidenciado desarticulaciones coxo-femorales, fémorotibiales y a nivel del tarso.

- La descarnación: hay numerosos restos que presentan señales de esta actividad, sobre todo coxales y diáfisis de huesos largos, donde la masa muscular es importante.

\section{ADQUISICIÓN DE LA PRESA: EL HOMBRE DE TAUTAVEL COMO CAZADOR DE MUFLONES}

La representación de los elementos anatómicos, la intensidad de la fragmentación, la casi ausencia de rastros de carnívoros y la presencia de numerosas marcas de corte, son los posibles elementos a favor de la aportación de los animales a la cueva por parte de los homínidos. La configuración espacial de los niveles arqueológicos asociados a la presencia de herramientas es, igualmente, prueba irrefutable de que las acumulaciones de huesos han sido depositadas antrópicamente (Villa et al., 1986).

Los homínidos aportaron los animales enteros desde el lugar de la matanza a su campamento, donde los desollaron y descuartizaron. Esto se manifiesta a partir del peso de los animales matados (alrededor de $150 \mathrm{Kg}$ ). No hay evidencias suficientes que nos permitan pensar en un primer tratamiento de los animales en el lugar de matanza. Por contra, según las características extrínsecas de los huesos, hemos podido descubrir que estos animales 
fueron desarticulados de maneras diversas. En esta fase es difícil saber si había una recuperación de la piel del animal. Hemos observado que, en numerosos casos, las señales son profundas, sin embargo, en otros, son netamente más discretas. Es probable que esta diferencia se deba a factores tales como la edad del animal o también podrían ser grados de rigidez. La habilidad del hombre carnicero es difícil de valorar. Es cierto, por otro lado, que la carne se recuperó y que todos los huesos largos, mandíbulas y primeras falanges, habían sido fracturados con el fin de extraer la médula. Numerosos fragmentos de huesos largos presentan señales de percusión (por ejemplo el $14 \%$ de tibias del nivel F). Éstas son marcas largas con líneas de fisuración (Villa et al., 1986 ; Blumenschine y Selvaggio, 1988).

Pero si la cueva de l'Aragó invita a pensar que para ciertos niveles hay una cierta especialización (por ejemplo para el nivel $\mathrm{F}$, con el muflón que representa el $63 \%$ de herbívoros), conviene, antes de todo, ser prudente con las conclusiones que se extraen. De hecho, la elección de las presas puede ser debida a criterios tales como la facilidad de caza, a una relación calidad/cantidad y también a criterios estacionales (repartición sexual). Hay que tener en cuenta que todos estos factores, junto a la naturaleza y composición de los grupos humanos y sus necesidades, el tipo de campamento y la duración de las ocupaciones nos son, por ahora, desconocidos. Estos últimos factores son los más corrientes, los que más se nos escapan, y los hábitos alimentarios corresponden a fenómenos complejos y variados, pero los datos arqueológicos que les conciernen son fragmentarios.

Aunque los primeros estudios tafonómicos muestran el mismo comportamiento del Hombre de Tautavel hacia otros animales (Moigne, 1983), es evidente que el estudio de una especie, en este caso el muflón, no es suficiente para comprender por sí solo las causas de la acumulación. Un análisis multiespecífico más complejo es necesario, cuando los datos precisos sobre los demás taxones, especialmente los cérvidos y los carnívoros, sean estudiados y publicados.

\section{AGRADECIMIENTOS}

Este artículo se ha podido realizar gracias al programa Human Capital and Mobility de la UE. Los resultados se han extraído de la tesis del autor, leída y defendida en la Université de la Méditerranée, Aix-Marseille II. Del mismo modo, debemos agradecer su ayuda al Profesor Henry de Lumley, a la Profesora Gloria Cuenca-Bescós, a Stephen y a todo el equipo del Àrea de Prehistòria de la Universitat Rovira i Virgili de Tarragona, y particularmente a Jordi Rosell, y al Profesor Eudald Carbonell.

\section{BIBLIOGRAFÍA}

Alhaique, F. 1994. Taphonomic analysis of the faunal remains from « $\mathrm{P}$ » and « $\mathrm{M}$ » layers of the Arena Candide (Savona, Italy). Quaternaria Nova, 4, 263-295.
Baryshnikov, G. 1989. Les mammifères du paléolithique inférieur du Caucase. L'Anthropologie, 93, 813-830.

Binford, L.R. 1978. Nunamiut Ethnoarchaeology. Academic Press, New-York, 504 pp.

Binford, L.R. 1981. Bones. Ancient men and Modern Myths. Academic Press, New-York, 320 pp.

Binford, L.R. 1984. Faunal remains from Klasies River Mouth. Academic Press, New-York, 294 pp.

Blasco Sancho, M.F. 1995. Hombres, Fieras y Presas. Estudio arqueozoológico y tafonómico del yacimiento del Paleolítico medio de la Cueva de Gabasa 1 (Huesca). Universidad de Zaragoza, Monografías Arqueológicas, 38, 205 pp.

Blumenschine, R.J. and Selvaggio, M.M. 1988. Percussion mark on bones surfaces as a new diagnostic of hominid behavior. Nature, 333, 763-765.

Blumenschine, R.J, Marean, C.W. and Capaldo, S.D. 1996. Blind test of interanalyst correspondance and accuracy in the identification of cut marks, percussion marks, and carnivore tooth marks on bone surface. Journal of Archaeological Science, 23, 493-507.

Bohlin, B. 1937. Eine tertiäre Saügertier-Fauna aus Tsaidam. Palaeontologia Sinica, 9, 1-111.

Bunn, H.T. 1982. Meat-eating and human evolution: studies on the diet and subsistence patterns of plio-pleistocene hominids in East-Africa. Philosophy Dissertation, University of California, Berkeley, 514 pp. (inédita).

Bunn, H.T. and Kroll, E. 1986. Systematic butchery by pliopleistocene hominids at Olduvai Gorge. Current Anthropology, 27, 431-452.

Capaldo, S.D. 1997. Experimental determinations of carcass processing by Plio-Pleistocene hominds and carnivores at FLK 22 (Zinjanthropus), Olduvai Gorge, Tanzania. Journal of Human Evolution, 33, 555-597.

Cattani, L., Cremaschi, M., Ferraris, M.R., Mallegni , M., Masini, F., Scola, V. et Tozzi, C. 1991. Le gisement du Pléistocène moyen de Visogliano (Trieste): restes humains, industries, environnement. L'Anthropologie, 95, 9-35.

Crégut, E. 1979. La faune des grands mammifères du gisement pleistocène moyen de la Caune de l'Arago à Tautavel (Pyrénées-Orientales). Travaux du Laboratoire de Paléontologie Humaine et Préhistoire, 3 tomes, Université de Provence, 391 pp. (inédita).

Crégut, E. 1980. La faune de mammifères du gisement pléistocène moyen anté-rissien de la Caune de l'Arago (Tautavel, Pyrénée-Orientales, France). Compte Rendu Académie des Sciences de Paris, 290, 751-754.

Crégut-Bonnoure, E. 1992. Dynamics of bovid migration in western Europe during the middle and late Pleistocene. Courier Forschungsinsitut Senckenberg, 153, 177-185.

Crégut-Bonnoure, E. 1995. Les petits bovidae de Venta Micena et de Cueva Victoria (Espagne). Congreso Internacional de Paleontologia Humana. Los Homindos y su Entorno en el Pleistoceno inferior y medio europeo, 3 a circular, 43.

Damuth, J. 1981. Population density and body size mammals. Nature, 290, 699-700.

Delpech, F. et Villa, P. 1993. Activités de chasse et de boucherie dans la grotte des Églises. Exploitation des 
Animaux sauvages à travers le temps. XIIe Rencontres Internationales d'Archéologie et d'Histoire d'Antibes, IVe Colloque International de l'Homme et l'Animal. Éd. ADPCA, 79-102.

Delpech, F., Le Tensorer, J.M., Pineda, R. et Prat, F. 1978. Un nouveau gisement pléistocène moyen : Camp-dePeyre à Sauveterre-la-Lémance (Lot-et-Garonne). Compte Rendu Académie des Sciences de Paris, 286, 1101-1103.

Díez, J.C. 1993. Zooarqueología de Atapuerca (Burgos) e implicaciones paleoeconómicas del estudio tafonómico de yacimientos del Pleistoceno Medio. Univ. Complutense de Madrid. Tesis Doctoral, 365 pp. (inédita).

Fosse, P. 1996. La grotte $n^{\circ} 1$ de Lunel-Viel (HéraultFrance): repaire d'hyènes du pléistocène moyen. Étude taphonomique du matériel osseux. Paléo, 8, 47-81.

Grayson, D.K. 1989. Bone transport, bone destruction, and reverse utility curves. Journal of Archaeological Science, 16, 643-652.

Guadelli, J.L. 1989. Etude taphonomique du repaire de Camiac (Gironde, France). Eléments de comparaison entre un site naturel et un gisement préhistorique. Bulletin de L'Association Française pour l'Etude du Quaternaire, 2, 91-100.

Huguet, R., Cáceres, L., Díez, J.C. y Rosell, J. (en prensa). Estudio tafonómico y zooarquelógico de los restos óseos de macromamíferos de la Unidad GII de Galeria (Sierra de Atapuerca, Burgos). In.: Atapuerca: Occupaciones Humanas y Paleoecologia del yacimiento de Galeria. Monografias Arqueologicas (Eds. E. Carbonell, A. Rosas y J.C Díez). Junta de Castilla y León.

Janossy, D. 1969. Stratigraphische Auswertung der europaïschen mittelpleistozänen Wirbelfauna. Berliner deutsche Geselleschaft geologische Wissenschaft. A. Geologie Paläontologie, 14, 573-643.

Jones, K.T. and Metcalfe, D. 1988. Bare bones in archaeology: bone marrow indices and efficiency. Journal of Archaeological Science, 15, 415-423.

Klein, R.G. 1982. Age (mortality) profiles as a means of distinguishing hunted species from scavenged ones in Stone Age archaeological sites. Paleobiology, 8, 151158.

Klein, R.G. and Cruz-Uribe, K. 1984. The analysis of animal bones from archaeological sites. University of Chicago Press, 266 pp.

Klein, R.G., Allawarden, K. and Wolf, C. 1983. The calculation and interpretation of ungulate age profiles from dental crown heights. In: Hunter-Gatherer economy in Prehistory: an European Perspective (Ed. G. Bailey). Cambridge University Press, 47-57.

Legendre, S. 1988. Les communautés de mammifères du Paléogène (Eocène supérieur et Oligocène) d'Europe occidentale: structures, milieux et évolution. Thèse de Doctorat d'État, Université des Sciences et Techniques du Languedoc, Montpellier, 265 pp. (inédita).

Levine, M.A. 1983. Mortality models and the interpretation of horse population structure. In: Hunter-Gatherer economy in Prehistory: an European Perspective (Ed. G. Bailey). Cambridge University Press, 23-46.
Lumley, H. de et Lumley, M.A. de. 1971. Découverte de restes humains anténéandertaliens datés du début du Riss à la Caune de l'Arago (Tautavel, Pyrénées-Orientales). Compte Rendu Académie des Sciences de Paris, 272, 1739-1742.

Lumley, H. de, Fournier, A., Park, Y.C., Yokoyama, Y. et Demouy, A. 1984. Stratigraphie du remplissage pleistocène moyen de la Caune de l'Arago à Tautavel. Étude de huit carottages effectués de 1981 à 1983. L'Anthropologie, 88, 5-18.

Lyman, R.L. 1984. Bone density and differential survivorship of fossil classes. Journal of Anthropological Archaeology, 3, 221-236.

Lyman, R.L. 1985. Bone frequencies: differential transport in situ destruction, and the MGUI. Journal of Archaeological Science, 12, 221-236.

Lyman, R.L. 1987a. On the analysis of vertebrate mortality profiles: sample size, mortality type, and hunting pressure. American Antiquity, 52, 125-142.

Lyman, R.L. 1987b. Archaeofaunas and butchery studies: a taphonomic perspective. Advances in Archaeological Method and Theory, 10, 249-337.

Lyman, R.L. 1992. Anatomical considerations of utility curves in zooarchaeology. Journal of Archaeological Science, 19, 7-22.

Lyman, R.L. 1994. Vertebrate taphonomy. Cambridge University Press, 524 pp.

Martinez, J.-N. 1991. L'astragale chez quelques artiodactyles du Néogène: morphologie comparée, morphométrie, aspect fonctionnel, intérêt systématique. D.E.A. de Paléontologie, option paléontologie des vertébrés, Université des Sciences et Techniques du Languedoc, Académie de Montpellier, 59 pp. (inédita).

Metcalfe, D. and Jones, K.T. 1988. A reconsideration of animal body-part utility indices. American Antiquity, 53, 486-504.

Miller, S.J. 1994. Biological agents of bone modification. Outillage peu élaboré en os et bois de cervidés IV: taphonomie/bone modification (Artéfacts 9), 67-75.

Moigne, A-M., 1983. Taphonomie des faunes quaternaires de la Caune de l'Arago à Tautavel. Thèse de troisième cycle, Université Pierre et Marie Curie, Paris, 344 pp. (inédita).

Monchot, H. 1996a. Les petits bovidés (genres Ovis, Hemitragus, Capra et Rupicapra) de la Caune de l'Arago (Tautavel, Pyrénées-Orientales). Thèse de Doctorat: Géologie du Quaternaire, Université de la Méditerranée, Aix-Marseille II, 433 pp. (inédita).

Monchot, H. 1996b. La consommation du mouflon (Ovis antiqua Pommerol, 1879) au pléistocène moyen à la Caune de l'Arago (Tautavel, Pyrénées-Orientales). Géologie Méditerranénne, 23, 101-115.

Monchot, H. 1997. La fragmentation des os longs du mouflon de la Caune de l'Arago (Tautavel, PyrénéesOrientales): une action anthropique. Paléo, 9, 55-67.

Moullé, P.E. 1992. Les grands mammifères du Pléistocène inférieur de la grotte du Vallonnet (Roquebrune-CapMartin, Alpes-Maritimes). Etude paléontologique des Carnivores, Equidé, Suidé et Bovidés. Thèse de Doctorat 
du Muséum National d'Histoire Naturelle, Paris, 365 pp. (inédita).

Olsen, S.L. and Shipman, P. 1988. Surface modification on bone: trampling versus butchery. Journal of Archaeological Science, 15, 535-553.

Palmqvist, P., Martínez-Navarro, B. y Arribas, A. 1996. Prey selection by terrestrial carnivores in a lower Pleistocene paleocommunity. Paleobiology, 22, 514-534.

Payne, S. 1973. Kill of patterns in sheep and goats: the mandible of Asvan Kale. Anatolian Studies, 23, 281-303.

Payne, S. 1987. Reference codes for wear states in the mandibular cheeck teeth of sheep and goats. Journal of Archaeological Science, 14, 609-614.

Pérez Ripoll, M. 1992. Marcas de carnicería, fracturas intencionadas y mordeduras de carnívoros en huesos prehistóricos del mediterráneo español. Colección Patrimonio, Istituto de Cultura «Juan Gil-Albert», Diputación provincial de Alicante, $269 \mathrm{pp}$.

Pernaud, J. 1990. Nouvelles données sur les cervidés de la Caune de l'Arago (Tautavel, Pyrénées-Orientales, France): étude des populations. Quaternaire, 3-4, 213223.

Pfeffer, F. 1967. Le mouflon de Corse (Ovis ammon musimon Schreber, 1782): position systématique, écologie et éthologie comparées. Mammalia, 31 supp., $262 \mathrm{pp}$.

Pommerol, F. 1879. Le mouflon quaternaire. Association Française Avancement des Sciences, 600-609.

Portis, A. 1918. Il rinventimento di "Ovis antiqua" Pommerol in teritorio di Roma. Bollettino Società Geologica Italiana, 36, 223-323.

Richardson, P.R.K. 1980. Carnivore damage on antelope bones and its archaeological implications. Paleontologia Africana, 23, 143-161.

Scott, K.M. 1983. Prediction of body weight of fossil artiodactyla. Zoological Journal of the Linnean Society, 77, 199-215.

Simonet, P. 1990. La faune des grands mammifères des couches supérieures du gisement de la Nauterie (La Romieu, Gers). Bulletin du Musée d'Anthropologie Préhistorique de Monaco, 53, 5-46.

Speth, J. 1983. Bison kills and bone counts. University of Chicago Press, 238 pp.

Stiner, M.C. 1990. The use of mortality patterns in archaeological studies of hominid predatory adaptations. Journal of Anthropological Archaeology, 9, 305-351.

Stiner, M.C. 1994. Honor among thieves. A zooarchaeological study of Neandertal Ecology. Princeton.

Teilhard de Chardin, P. et Piveteau, J. 1930. Les mammifères fossiles du Nihowan (Chine). Annales Paléontologie, 19, 1-134.

Trolle-Lassen, T. 1990. Butchering of red deer (Cervus elaphus, L.). A study from the late mesolithic settlment of Tybrind Vig, Denmark. Journal of Danish Archaeology, 9, 7-17.

Valensi, P. 1991. Étude des stries de boucherie sur les ossements de cerf des niveaux supérieurs de la Grotte du Lazaret (Nice, Alpes-Maritimes). L'Anthropolgie, 95, 797-830.

Valensi, P. 1996. Taphonomie des grands mammifères et palethnologie des niveaux supérieurs à la grotte du Lazaret (Nice, France). Anthropozoologica, 23, 13-28.

Villa, P. and Mahieu, É. 1991. Breakage patterns of human long bones. Journal of Human Evolution, 21, 27-48.

Villa, P., Courtin, J., Helmer, D., Shipman, P., Bouville, C. et Mahieu, É. 1986. Un cas de cannibalisme au néolithique. Boucherie et rejet de restes humains et animaux dans la grotte de Fontbregoua à Salernes (Var). Gallia Préhistoire, 29,143-171.

Villaverde, V., Martínez -Valle, R., Guillem, P.M. y Fumanal, M.P. 1996. Mobility and the role of small game in the Middle Paleolithic of the central region of the spanish mediterranean: a comparison of Cova Negra with other paleolithic deposits. In: The last Neandertals, the first anatomically modern Humans (Eds. E. Carbonell and M.Vaquero). 267-288. 